\title{
Article \\ A Generalized Fractional Power Series for Solving a Class of Nonlinear Fractional Integro-Differential Equation
}

\author{
Sirunya Thanompolkrang ${ }^{1}$ and Duangkamol Poltem ${ }^{1,2, *}$ \\ 1 Department of Mathematics, Faculty of Science, Burapha University, Chonburi, 20131, Thailand; \\ 59910295@go.buu.ac.th \\ 2 Centre of Excellence in Mathematics, Commission on Higher Education, Ministry of Education, Bangkok \\ 10400, Thailand \\ * Correspondence: duangkamolp@buu.ac.th; Tel.: +6-638-103-099
}

Version September 25, 2018 submitted to Preprints

\begin{abstract}
In this paper, we investigate an analytical solution of a class of nonlinear fractional integro-differential equation base on a generalized fractional power series expansion. The fractional derivatives are described in the conformable's type. The new approach is a modified form of the well-known Taylor series expansion. The illustrative examples are presented to demonstrate the accuracy and effectiveness of the proposed method.
\end{abstract}

Keywords: fractional power series; integro-differential equations; conformable derivative

\section{Introduction}

Fractional calculus and fractional differential equations are widely explored subjects thanks to the great importance of scientific and engineering problems. For example, fractional calculus is applied to model the fluid-dynamic traffics [1], signal processing [2], control theory [3], and economics [4]. For more details and applications about fractional derivative, we refer the reader to [5-8]. Many mathematical formulations contain nonlinear integro-differential equations with fractional order. However, the integro-differential equations are usually difficult to solve analytically, so it is required to obtain an efficient approximate solution. For instance we can mention the following papers. Rawashdeh [9] applied collocation method to study the integro-differential equations of fractional order, authors of [10] applied spectral collocation method to solve stochastic fractional integro-differential equations. Momani [11] applied the Adomian decomposition method (ADM) to approximate solutions for fourth-order integro-differential equations of fractional order. Nawaz [12] applied the variational iteration method and homotopy perturbation method for the fourth-order fractional integro-differential equations, authors of [13] presented a computational method based on the second kind Chebyshev wavelet to solve fractional nonlinear Fredholm integro-differential equations. In Ref. [14] approximated solution of fractional integro-differential equations by Taylor expansion method. Among these methods, the Taylor expansion method is more attractive. Hitherto several fractional power series expansions have been presented in the literature [15-21]. In Ref. [18] the authors presented a new algorithm for obtaining a series solution for a class of fractional differential equations. Syam [19] investigate a numerical solution of fractional Lienard's equation by using the residual power series method. In Ref. [20] the authors develop a new method to solve rational or irrational order fractional differential equations. This method is called the restricted fractional differential transform method (RFDTM). Recently, Jaradat [21] proposed a new method based on Taylor series expansion for solving the fractional (integro)-differential equations and compared numerical solution with exact solution. A new series expansion is proposed to obtain closed-form solutions of the fractional (integro-)differential equations in the Caputo's type. This expansion provide a more integrated representation of the fractional power series with a related convergence theorem called a generalized fractional power series (GFPS). 
In this paper, we adopt the conformable fractional derivative with GFPS and apply it to solve a class of nonlinear integro-differential equation

$$
T^{\alpha}[y(t)]=h(t)+\int_{0}^{1} k(t, \tau)[y(\tau)]^{q} d \tau, \quad q \geq 1,
$$

subject to the initial conditions

$$
y^{(i)}(0)=a_{i}
$$

${ }_{45} \alpha \in(0,1]$.

Definition 1. Given a function $f:[0, \infty) \rightarrow \mathbb{R}$, where the conformable fractional derivative of $f$ order $\alpha$ is defined by

$$
T^{\alpha}[f(t)]=\lim _{\varepsilon \rightarrow 0} \frac{f\left(t+\varepsilon t^{1-\alpha}\right)-f(t)}{\varepsilon}
$$

46 for all $t>0$.

Theorem 1. If $f$ and $g$ be $\alpha$-differentiable at a point $t>0$, then

$$
T^{\alpha}[a f+b g]=a T^{\alpha}[f]+b T^{\alpha}[g]
$$

47 for all $a, b \in \mathbb{R}$.

4

The power rule of the conformable fractional derivative is given as follows.

Theorem 2. The conformable fractional derivative of the power function is given by

$$
T^{\alpha}\left[t^{p}\right]=p t^{p-\alpha}
$$

49 for all $p \in \mathbb{R}$.

We implement the generalized fractional power series (GFPS) [21] to solve Equation (1). We start 51 by the following definition and some related properties to the GFPS.

Definition 2. A generalized fractional power series of the form

$$
\sum_{i+j=0}^{\infty} c_{i j} t^{i \alpha+j}=c_{00}+c_{01} t^{1}+c_{10} t^{\alpha}+c_{02} t^{2}+c_{11} t^{\alpha+1}+c_{20} t^{2 \alpha}+\cdots
$$

52 where $t>0$, is called generalized fractional power series (GFPS) about $t=0 . c_{i j}$ denote the coefficients of the 53 series, where $i, j \in \mathbb{N}$. 
Moreover, the GFPS is naturally obtained as a Cauchy product of two power series, as following

$$
\sum_{i+j=0}^{\infty} c_{i j} t^{i \alpha+j}=\left(\sum_{i=0}^{\infty} a_{i} t^{i \alpha}\right)\left(\sum_{j=0}^{\infty} b_{j} t^{j}\right),
$$

54 where $c_{i j}=a_{i} b_{j}$.

55 Proposition 1. If $\sum_{k=0}^{\infty} a_{k} t^{k \alpha}$ converges for some $t=a>0$, then it converges absolutely for $t \in(0, a)$.

56 Proof. See [21].

57 Corollary 1. If $\sum_{k=0}^{\infty} b_{k} t^{k}$ converges for some $t=b>0$, then it converges absolutely for $t \in(0, b)$.

58 Proof. See [21].

59 Theorem 3. Consider the two power series $A=\sum_{k=0}^{\infty} a_{k} t^{k \alpha}$ and $B=\sum_{k=0}^{\infty} b_{k} t^{k}$ such that $A$ converges 6o absolutely to a for $t=t_{a}>0$ and $B$ converges to $b$ for $t=t_{b}>0$. Then the Cauchy product of $A$ and $B$ 61 converges to ab for $t=t_{c}>0$ where $t_{c}=\min \left\{t_{a}, t_{b}\right\}$.

62 Proof. See [21].

\section{3. The Generalized Conformable Fractional Power Series Method}

In this section, we present a generalized conformable fractional power series method to solve problem (1) and (2). We assume that the solution $y(t)$ takes the form

$$
y(t)=\sum_{i+j=0}^{\infty} c_{i j} t^{i \alpha+j}
$$

64 where $y(0)=y_{0}$ and $c_{i j}$ are constants to be determined. Clearly, $c_{00}=y_{0}$.

${ }_{65}$ Theorem 4. The generalized conformable fractional power series (GCFPS) of order $\alpha$ is given by

$$
\begin{aligned}
T^{\alpha}[y(t)] & =\sum_{i=1}^{\infty} c_{i 0}(i \alpha) t^{(i-1) \alpha}+\sum_{j=1}^{\infty} c_{0 j}(j) t^{j-\alpha} \\
& +\sum_{i+j=0}^{\infty} c_{i+1, j+1}[(i+1) \alpha+j+1] t^{i \alpha+j+1},
\end{aligned}
$$

66 where $t$ be a positive real number.

Proof. Assuming we can interchange the summation and the fractional derivative operator and using Theorems 1 and 2, by term-by-term differentiation within the interval of convergence of $t>0$, if

$$
y(t)=\sum_{i+j=0}^{\infty} c_{i j} t^{i \alpha+j}
$$

then we obtain Equation (7).

The proposed GCFPS expansion (7) will be utilized to introduce a parallel scheme to the power series solution method. The illustrative examples are presented to demonstrate the accuracy and effectiveness of the proposed method in Section 4. 


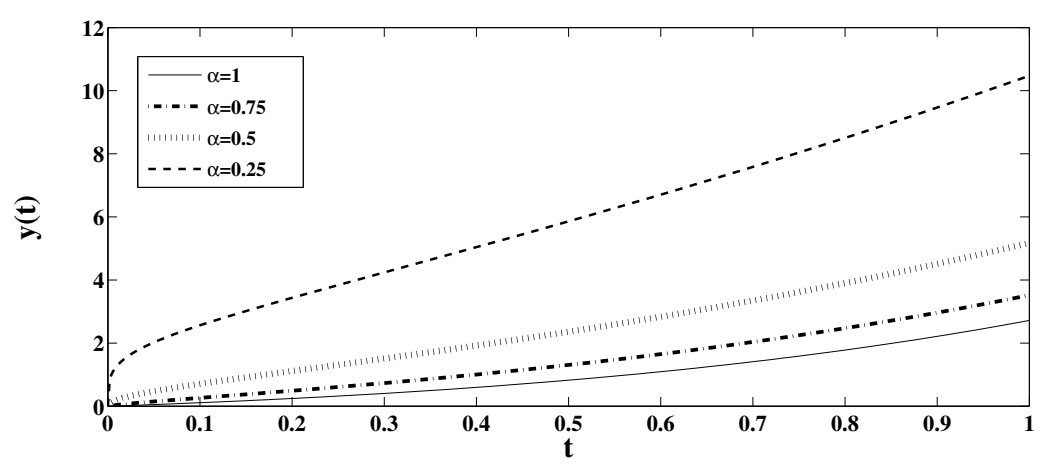

Figure 1. The approximate solution of Example 1 for $\alpha=0.25,0.5,0.75,1$.

Example 1. Consider the nonlinear Fredholm fractional integro-differential equation

$$
T^{\alpha}[y(t)]=t e^{t}+e^{t}-t+\int_{0}^{1} t y(\tau) d \tau, 0 \leq t<1,0<\alpha \leq 1
$$

75 subject to the initial condition $y(0)=0$.

In the previous discussion and using the initial condition, the proposed generalized fractional power series solution to Equation (8) has the form

$$
y(t)=\sum_{i+j=1}^{\infty} c_{i j} t^{i \alpha+j}
$$

By substituting Equation (9) into Equation (8), the coefficients $c_{i j}, i+j \geq 1$ are determined by equating the coefficients of like powers of $t$ through determining a formal recurrence relation. We have obtained

$$
\begin{gathered}
c_{11}=\frac{\alpha+2}{\alpha^{2}+3 \alpha+1}\left[\frac{\alpha(\alpha+1)+1}{\alpha(\alpha+1)}+\sum_{j=2}^{\infty} \frac{j+1}{(\alpha+j)(\alpha+j+1) j !}\right], \\
c_{1 j}=\frac{j+1}{(\alpha+j) j !} \text { for } j=0,2,3,4, \ldots,
\end{gathered}
$$

and $c_{i j}=0$ otherwise. Therefore, the exact solution of Equation (8) is

$$
y(t)=c_{11} t^{\alpha+1}+\frac{t^{\alpha}}{\alpha}+\sum_{j=2}^{\infty} \frac{j+1}{(\alpha+j) j !} t^{\alpha+j},
$$

with $c_{11}$ as in Equation (10). Particularly with $\alpha=1$, it can be obtained that the exact solution for the classical version of Equation (8) is

$$
y(t)=t^{2}+t+\sum_{j=2}^{\infty} \frac{t^{j+1}}{j !}=t e^{t} .
$$

76 Figure 1 illustrates the approximate solutions for $\alpha=0.25,0.5,0.75,1$ in $I \in[0,1)$. 


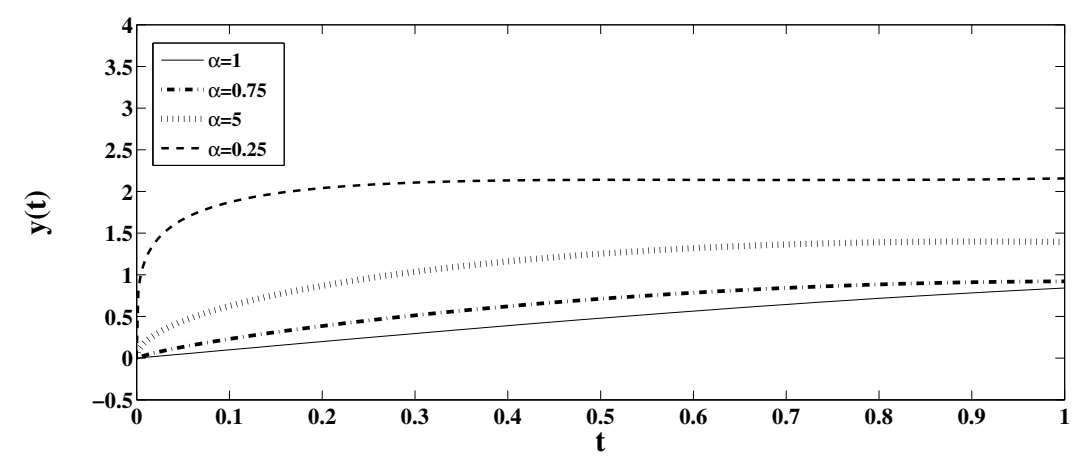

Figure 2. The approximate solution of Example 2 for $\alpha=0.25,0.5,0.75,1$.

Example 2. Consider the Volterra integro-differential equation:

$$
T^{\alpha}[y(t)]=1-\int_{0}^{t} y(\tau) d \tau, 0 \leq t \leq 1,0 \leq \alpha \leq 1, y(0)=0 .
$$

Upon substituting all the relevant quantities into the Equation (14) and collecting powers of $t$, we have

$$
\begin{gathered}
c_{10}=\frac{1}{\alpha}, \\
c_{i+1, i}=\frac{1}{\alpha}\left[\frac{(-1)^{i}}{(\alpha+1)(2 \alpha+1) \cdots(i \alpha+i)((i+1) \alpha+i)}\right] \text { for } i=1,2,3, \ldots,
\end{gathered}
$$

where $c_{i j}=0$ otherwise. Then, the exact solution is

$$
y(t)=\frac{1}{\alpha} t^{\alpha}+\sum_{i=1}^{\infty} c_{i+1, i} t^{(i+1) \alpha+i}
$$

77 where $c_{i+1, i}$ satisfies Equation (16).

78 Particularly, we can see the approximate solutions for $\alpha=1$ which are derived for different values of $t$.

79 Then, the exact solution in a closed form $y(t)=\sin t$. Figure 2 shows the effect of $\alpha$ on the solution for

so $\alpha=0.25,0.5,0.75,1$ in $I \in[0,1)$.

Example 3. Consider the nonlinear Fredholm fractional integro-differential equation

$$
T^{\frac{1}{2}}[y(t)]=2 t^{\frac{3}{2}}-t^{\frac{1}{2}}-\frac{t}{1260}+\int_{0}^{1} t \tau[y(\tau)]^{4} d \tau
$$

s1 subject to the initial condition $y(0)=0$.

Since the definite integral in Equation (18) completely depends on the variable $\tau$, the solution is spanned by the monomials $\left\{t, t^{\frac{3}{2}}, t^{2}\right\}$. That is

$$
y(t)=c_{01} t+c_{11} t^{\frac{3}{2}}+c_{02} t^{2}
$$

with

$$
T^{\frac{1}{2}}[y(t)]=c_{01} t^{\frac{1}{2}}+\frac{3}{2} c_{11} t+2 c_{02} t^{\frac{3}{2}}
$$

Substituting all the relevant quantities into the Equation (18) and equating the coefficients of like powers of $t$ from both sides, we obtain $c_{01}=-1, c_{02}=1$, and $c_{11}$ satisfies

$$
c_{11}\left(c_{11}^{3}-\frac{128}{255} c_{11}^{2}+\frac{4}{21} c_{11}-\left(\frac{1024}{20995}+12\right)\right)=0 .
$$


Subsequently, we have exact solutions in the form $y(t)=t^{2}-t+c_{11} t^{\frac{3}{2}}$ where $c_{11}$ satisfies Equation (21).

\section{Conclusions}

In this paper, we have investigated the analytical solution of a class of nonlinear integro-differential equation based on the GCFPS method. Three examples of our numerical results are presented. From Figures 1 and 2, we see that as $\alpha$ is increasing, the approximate solution is decreasing. The results reveal that the exact solutions are obtained in the form of a rapidly convergent series with an easily computable component. In conclusion, the proposed scheme could be used further in studying identical applications. It can be extended to solve a variety of fractional differential and integral equations in sciences and engineering.

Author Contributions: Both the authors have contributed equally in the article

Funding: This research was funded by Burapha University through National Research Council Thailand grant no. 173/2561 and Science Achievement Scholarship of Thailand.

Acknowledgments: The authors are grateful to the reviewers for valuable suggestions in improving the quality of the paper.

Conflicts of Interest: The authors declare no conflicts of interest.

\section{Abbreviations \\ The following abbreviations are used in this manuscript: \\ RFDTM Differential transform method \\ GFPS Generalized fractional power series \\ GCFPS Generalized conformable fractional power series}

\section{References}

1. He, J.H. Some applications of nonlinear fractional differential equations and their approximations. Bull. Sci. Technol. 1999, 15, 86-90.

2. Panda, R.; Dash, M. Fractional generalized splines and signal processing. Signal Process 2006, 86, 2340-2350.

3. Bohannan, G.W. Analog fractional order controller in temperature and motor control application. J. Vib. Control 2008, 14, 1487-1498.

4. Baillie, R.T. Long memory processes and fractional integration in econometrics. J. Econometrics 1996, 73, 5-59.

5. Samko, G.; Kilbas, A.A.; Marichev. Fractional Integrals and Derivatives: Theory and Applications, Gordon and Breach, Yverdon, 1993.

6. Kilbas, A.; Srivastava, M.H.; Trujillo, J.J. Theory and Application of Fractional Differential Equations, Amsterdam, Elsevier, 2006.

7. Diethelm, K. The analysis of fractional differential equations, New York, Springer, 2010.

8. Caponetto, R.; Dongola, G,; Fortuna, L.; Petras, I.Fractional order systems: mod- eling and control applications, Singapore, World Scientific, 2010.

9. Rawashdeh, E.A. Numerical solution of fractional integro-differential equations by collocation method. Appl. Math. Comput. 2006, 176, 1-6.

10. Taheri, Z.; Javadi, S.; Babolian, E. Numerical solution of stochastic fractional integro-differential equation by the spectral collocation method. J. Comput. Appl. Math. 2017, 321, 336-347.

11. Momani, S.; Noor, M. A. Numerical methods for fourth order fractional integro-differential equations. Appl. Math. Comput. 2006, 182, 754-760.

12. Nawaz, Y. Variational iteration method and homotopy perturbation method for fourth-order fractional integro-differential equations. Comput. Math. Appl. 2011, 61, 2330-2341.

13. Zhu, L.; Fan, Q. Solving fractional nonlinear Fredholm integro-differential equations by the second kind Chebyshev wavelet. Commun. Nonlinear Sci. Numer. Simulat. 2012, 17, 2333-2341.

14. Huang, L.; Li, X.F.; Zhao, Y.; Duan, X.Y. Approximate solution of fractional integro-differential equations by Taylor expansion method. Comput. Math. Appl. 2011, 62, 1127-1134. 
15. El-Ajou, A.; Abu Arqub, O.; Al Zhour, Z.; Momani, Z. New results on fractional power series: theories and applications. Entropy 2013, 15, 5305-5323.

16. El-Ajou, A.; Abu Arqub, O.; Al-Smadi, M.A. A general form of the generalized taylors formula with some applications. Appl. Math. Comput. 2015, 256, 851-859.

17. Odibat, Z.; Shawagfeh, N. Generalized taylors formula. Appl. Math. Comput. 207, 186, $286-293$.

18. Al-Refai, M.; Hajji, M.A.; Syan, M.I. An efficient series solution for fractional differential equation. Abstr. Appl. Anal. 2014, doi:10.1155/2014/891837.

19. Syan, M.I. A numerical solution of fractional Lienard's equation by using the residual power series method. Comput. Math. Appl. 2018, 6, doi:10.3390/math6010001.

20. Khudair, A.R; Haddad, S.A.M.; Khalaf, S.L. Restricted fractional differential transform for solving irrational order fractional differential equations. Chaos Solitons Fract. 2017, 101, 81-85.

21. Jaradat, I.; Al-Dolat, M.; Al-Zoubi, K.; Alquran, M. Theory and applications of a more general form for fractional power series expansion. Chaos Solitons Fract. 2017, 108,107-110.

22. Khalil, R.; Horani, M.A.; Yousef, A.; Sababheh, M. A new definition of fractional derivative. J. Comput. Appl. Math. 2014, 264, 65-70. 